\title{
Research on maintenance method of relay protection state based on combination weight and gray relation
}

\author{
Xuanhang Wang, and Zhijian Liang* \\ College of Electrical Engineering, Guangxi University, China
}

\begin{abstract}
Relatively independent evaluation parameters are selected from many parameters through pedigree clustering.Learning the analytic hierarchy process (ahp) and entropy weight method can determine the weight, and at the same time to understand the error of the analytic hierarchy process (ahp) and entropy weight method is large, so the combination of the subjective and objective weight obtained by the two methods, using the improved entropy weight-ahp method to determine the weight.The improved weight calculation method has a clear hierarchical structure, which not only considers the influence of subjective and objective factors, but also makes full use of the weight information in the hierarchical structure.Considering the uncertainty of information, gray relation is adopted to deal with the data, so as to make maintenance rules.
\end{abstract}

\section{Introduction}

Combining the experience in power grid maintenance work site, repairing the power grid requires timeliness, in excess of the prescribed time limit to repair unfinished tasks, will be the inspection, repair often run a day when many substation hair stand, but still can't finish the repair, especially in the summer peaks, flood control and buy electric and other major electrical tasks, requirements to repair timely.Now the society needs to ensure the stability of electric energy, so, when the failure occurs, need to complete the repair task within the deadline, overdue, repair personnel will be assessed.Therefore, it is very important to put forward a more scientific and effective state maintenance scheme to reduce the operation cost of power grid and improve and ensure the power quality.Table 1. Setting Word's margins.

\section{The Importance of relay protection}

Now electric energy has been widely used in every industry, production and living of human society into the age of electricity power is the most important in the modern society is the most convenient sources of energy, are the tools of scientific development to transfer production distribution and consumption of electricity power system of electric equipment

* Corresponding author: 916328128@,qq.com 
together, forming a whole to ensure that the power balance between supply and demand so safe and reliable operation of power system is the necessary condition of social and economic development is an important guarantee of maintaining social stability, is also the purpose of power system operation Relay protection system for power system operation may occur in the process of all kinds of faults and abnormal running state, make reasonable measures to protect the safe and stable operation of power system with the development of smart grid.

\section{Traditional maintenance program}

The relay protection system is an important part of the safe and reliable operation of the power system. If the relay protection system is defective, it may affect the reliable operation of the system. Therefore, the elimination of the relay protection system is an indispensable part of daily work The traditional maintenance scheme has the following disadvantages: The so-called state maintenance, also referred to as CBM, is mainly based on the advanced state monitoring and evaluation technology to obtain the relevant data to evaluate the operation of equipment, equipment potential failure warning, as far as possible before the equipment failure implementation of the corresponding maintenance mode.Based on the current operation situation of related equipment, CBM evaluates the potential fault location, fault degree and development direction of related equipment through various fault occurrence signals identified in advance by leading monitoring methods, and then develops appropriate maintenance strategies to guide the smooth development of maintenance work. According to relevant data, after state maintenance, the failure rate of related facilities is reduced by $75 \%$, and the overall maintenance cost is correspondingly reduced by $30 \%$ to $50 \%$.Table 2. Formatting sections, subsections and subsubsections.

\section{The research method adopted}

\subsection{Data collection phase}

At present, the main data available online are dielectric loss, leakage current, partial discharge and so on.It is difficult to carry out on - line monitoring of conventional relay protection equipment.The reason is that the secondary circuit is composed of a large number of relays and connection cables, with multiple and scattered points. It is not only technically difficult but also uneconomical to realize online monitoring of the wiring state of relays and circuits.

\subsection{Positioning}

Cluster analysis, also known as group analysis, is a multivariate statistical method for the quantitative classification of samples or indicators.It divides a group of sample vectors in the feature space into several subsets according to some similarity criteria, so that similar samples are classified into one category and dissimilar samples into different categories. Genealogical clustering analysis is to aggregate the high degree of similarity into a small category according to the similarity coefficient between various research objects, and then gradually expand to aggregate the alienation into a large category.For evaluation parameters, the purpose is to screen out relatively independent parameters from many parameters for evaluation Before calculation, it is necessary to deal with the original data to 
eliminate the difference between dimensions. The normalization method is commonly used, and the calculation formula is

$$
x^{\prime}=\frac{x-x_{\min }}{x_{\max }-x_{\min }}
$$

where, xmax represents the maximum value of parameter $\mathrm{x}$, and xmin represents the minimum value of parameter $x$. Distance coefficient is a classification statistic commonly used in cluster analysis, which is used to represent the similarity between research objects.Is expressed as

$$
\mathrm{d}_{(x, y)}=\sqrt{\sum_{i=1}^{n}\left(x_{i}-y_{i}\right)^{2}}
$$

Relative independent evaluation parameters were selected according to cluster analysis pedigree diagram.

\subsection{Determination of combined weight of state comprehensive evaluation index system of relay protection device}

\subsubsection{Improved entropy weight hierarchy method}

The analytic hierarchy process (ahp) decomposes the complex decision-making problem into multiple layers, such as target layer, criterion layer and scheme layer.Because the analytic hierarchy process (ahp) theory is clear, simple and practical, it is widely used in multi-objective decision-making.Entropy weight method introduces thermodynamic theory into mathematical statistics. Although it avoids the influence of subjective factors to a certain extent, the relative weight cannot be completely equal to the actual importance. Therefore, errors may occur.Since both the analytic hierarchy process (ahp) and the entropy weight method have large errors, it can be considered to combine the subjective and objective weights obtained by the two methods.

\subsubsection{Specific steps}

(1) The events to be evaluated are taken as the top layer, the criteria for evaluating the events as the middle layer, and the various state quantities collected as the bottom layer.Let's say I have B elements in my middle layer,there are $\mathrm{n}$ elements at the bottom,Each middle layer element contains $\mathrm{n}$ states.

(2) Entropy weight method is used to calculate the objective weight of the lowest element

$$
A=\left\{\alpha_{1}, \alpha_{2}, \cdots \alpha_{\mathrm{n}}\right\}
$$

(3) The weight of the middle layer element is obtained by using the method of ahp to calculate the subjective weight 


$$
\beta=\left\{\beta_{1}, \beta_{2}, \cdots \beta_{\mathrm{m}}\right\}
$$

The weight of the lowest level element is

$$
\varphi=\left\{\varphi_{1}, \varphi_{2}, \cdots, \varphi_{n}\right\}
$$

(4) The comprehensive weight of entropy weight hierarchy method is obtained by synthesizing the subjective weight and objective weight of the lowest element

$$
\tau=\left\{\tau_{1}, \quad \tau_{2}, \cdots, \tau_{n}\right\}
$$

According to the corresponding relationship between the middle layer and the bottom layer, the comprehensive weight of the bottom layer is expressed as

$$
\tau=\left\{\tau_{11}, \tau_{12}, \cdots \tau_{1 n}, \tau_{21}, \tau_{22}, \cdots \tau_{2 n}, \cdots, \tau_{m 1}, \tau_{m 2}, \cdots, \tau_{m n}\right\}
$$

The results were normalized respectively

$$
\Omega=\left\{\omega_{11}, \omega_{12}, \cdots \omega_{1 n}, \omega_{21}, \omega_{22}, \cdots \omega_{2 n}, \cdots, \omega_{m 1}, \omega_{m 2}, \cdots \omega_{m n}\right\}
$$

The middle layer weight is multiplied by the comprehensive weight to get the weight

$$
\Omega=\left\{\omega_{1}, \omega_{2}, \cdots, \omega_{n}\right\}
$$

The improved weight calculation method has a clear hierarchical structure, which not only considers the influence of subjective and objective factors, but also makes full use of the weight information in the hierarchical structure.

\subsection{Gray correlation}

Starting from the incomplete information, the gray relational degree method reflects the degree of similarity between the evaluation objects and the reference objects by comparing the numerical distribution characteristics between the sequences, and thus obtains the order of advantages and disadvantages.

(1) Establish the decision matrix and standardized processing first. Suppose there are $n$ evaluation objects and $\mathrm{m}$ indicators, and the corresponding original data of each indicator is

$x_{i j}(1 \leq i \leq n, 1 \leq j \leq m)$, Establish the initial decision matrix $X=\left(x_{i j}\right)_{n \times m}$, Since the attributes and dimensions of each index are not comparable, the original index is standardized. $r_{i j}=\left(x_{i j}-\min x_{i j}\right) /\left(\max x_{i j}-\min x_{i j}\right)$

After the original matrix $\mathrm{X}$ is standardized, the standardized decision matrix is obtained

$$
R=\left(r_{i j}\right)_{n \times m} r_{i j} \in[0,1]
$$

The optimization of each index can be obtained

$$
r_{* j}=\max r_{i j}
$$


And the virtual ideal solution

$$
R_{*_{j}}=\left[r_{*_{1}}, r_{*_{2}}, \cdots, r_{*_{m}}\right]
$$

(2) Calculate gray correlation coefficient and gray correlation degree. The gray correlation coefficient between the ith evaluation object and the ideal solution on the jTH index is calculated as

$$
\varepsilon_{i j}=\frac{\min _{i \in n} \min _{i \in m}\left|r_{*_{j}}-r_{i j}\right|+\rho \max _{i \in n} \max _{i \in m}\left|r_{*_{j}}-r_{i j}\right|}{\left|r_{*_{j}}-r_{i j}\right|+\rho \max _{i \in n} \max _{j \in m}\left|r_{*_{j}}-r_{i j}\right|}
$$

The gray correlation coefficient matrix between the evaluation object and the ideal solution is established as

$$
R^{+}=\left[\varepsilon_{i j}\right]_{n \times m}
$$

Introduce the weight vector and calculate the gray relational degree between the ith evaluation object and the ideal solution as

$$
G_{i}=\sum_{j=1}^{m} \omega_{j} \varepsilon_{i j}(i=1,2, \cdots, n)
$$

\section{Conclusion}

According to the gray relational degree calculated, the severity of the fault can be screened and sorted based on the comprehensive factors.Based on the known standardized decision matrix, the gray correlation analysis method is used to calculate the correlation coefficient and the correlation degree of each fault.

This study was conducted under the guidance of associate professor Zhijian Liang from the school of electrical engineering of guangxi university, with the help of his classmates and the generous help of his grid colleagues.

\section{References}

1. Ren H, Xu Y, Zhou Q J. Research on Condition Evaluation Method of Relay Protection Based on Reliability Indices[J]. Applied Mechanics \& Materials, 2013, 373375:1509-1513.

2. Liu Z N, Xiao S W, Jiang S. Research on Evaluation Method of Relay Protection Action Based on Fault Information[J]. Advanced Materials Research, 2014, 10441045:490-494.

3. Zhang Y, Qin J, Wang Q, et al. Research on maintenance decision-making method of relay protection based on interval numbers grey fuzzy comprehensive evaluation[J]. Power System Protection \& Control, 2014, 42(4):16-21.

4. Qian L. Evaluation and management system of Zhejiang Grid relay protection equipments[J]. Computer Era, 2012. 
5. Huang S F, Chen S Y, Qiu Y T, et al. Online condition monitoring methodology for relay protection based on self-test information[C]// International Conference on Advanced Power System Automation and Protection. IEEE, 2012:256-260.

6. Zi-Li X U, Liao H X, Yuan X Q, et al. Design and Implementation of Relay Protection On-Line Monitoring and Evaluation Management System[J]. East China Electric Power, 2014. 